\title{
Reactivity and Selectivity in Catalytic Reactions of Enoldiazoacetamides. Assessment of Metal Carbenes as Intermediates.
}

\author{
Yongming Deng, ${ }^{\dagger}$ Changcheng Jing, ${ }^{\dagger}$ Hadi Arman, ${ }^{\dagger}$ Michael P. Doyle ${ }^{\dagger *}$ \\ ${ }^{\dagger}$ Department of Chemistry, The University of Texas at San Antonio, San Antonio, Texas \\ 78249, United States \\ tShanghai Engineering Research Center of Molecular Therapeutics and New Drug \\ Development, East China Normal University, Shanghai, 200062, P. R. China
}

\section{Supporting Information}

S1 Figure S1. Intramolecular reaction of enoldiazoacetamide 1a catalyzed by $\mathrm{ZnCl}_{2}$ through its cyclopropene intermediate $\mathbf{2} \mathbf{a}$ to form $\mathbf{3 a}$ and $\mathbf{4 a}$ monitored by ${ }^{1} \mathrm{H}$ NMR spectroscopy.

S1 Figure S2. Intramolecular reaction of enoldiazoacetamide 1a catalyzed by $\mathrm{Cu}(\mathrm{MeCN})_{4} \mathrm{PF}_{6}$ through its cyclopropene intermediate 2a to form 3a and 4a monitored by ${ }^{1} \mathrm{H}$ NMR spectroscopy.

S2 Figure S3. Intramolecular reaction of enoldiazoacetamide $\mathbf{1 a}$ catalyzed by $[\operatorname{Ru}(p$ cymene) $\left.\mathrm{Cl}_{2}\right]_{2}$ to form $\mathbf{3 a}$ monitored by ${ }^{1} \mathrm{H}$ NMR spectroscopy.

S2 Figure S4. Intramolecular reaction of donor-acceptor cyclopropene $\mathbf{2 a}$ catalyzed by $\left[\mathrm{Co}\left(3,5-\mathrm{Di}{ }^{t} \mathrm{Bu}-\mathrm{IbuPhyrin}\right)\right]$ to form 3a monitored by ${ }^{1} \mathrm{H}$ NMR spectroscopy.

S3-S5 X-Ray Diffraction Analysis for Compound 6.

S6-S9 ${ }^{1} \mathrm{H}$ NMR and ${ }^{13} \mathrm{C}$ NMR Spectra of $4 \mathrm{~b}, 3 \mathrm{~d}, 4 \mathrm{~d}, 6$ 

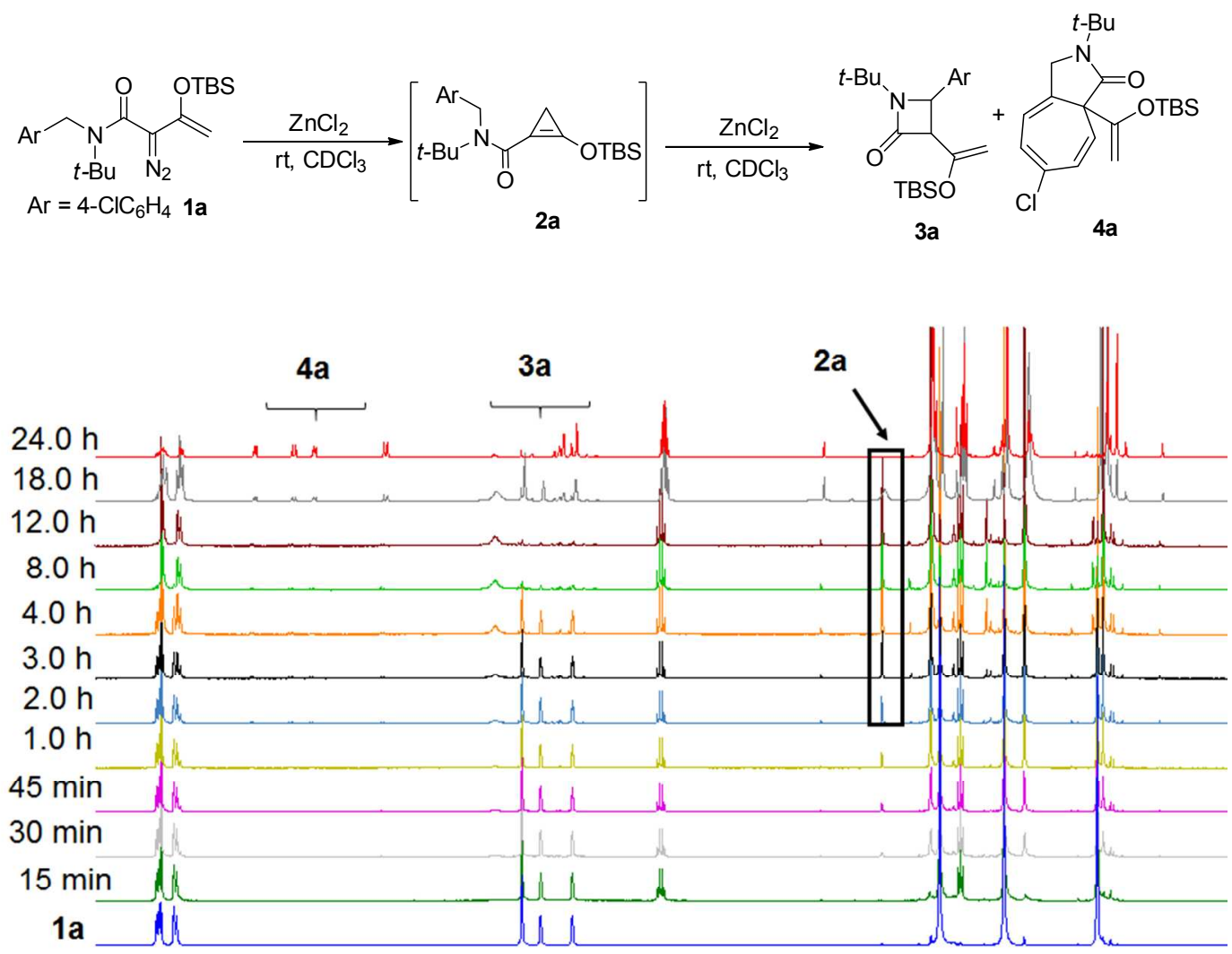

Figure S1. Intramolecular reaction of enoldiazoacetamide 1a catalyzed by $\mathrm{ZnCl}_{2}$ through its cyclopropene intermediate $\mathbf{2 a}$ to form $\mathbf{3 a}$ and $\mathbf{4 a}$ monitored by ${ }^{1} \mathrm{H}$ NMR spectroscopy.

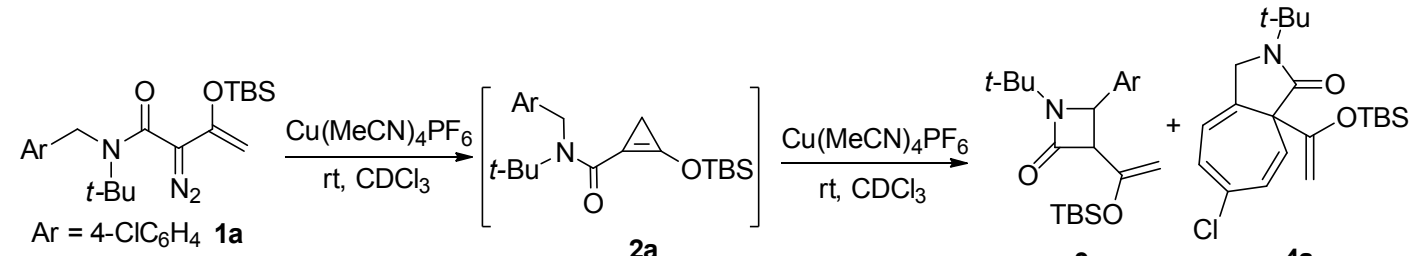
$\mathrm{Ar}=4-\mathrm{ClC}_{6} \mathrm{H}_{4} 1 \mathrm{a}$

$4 a$

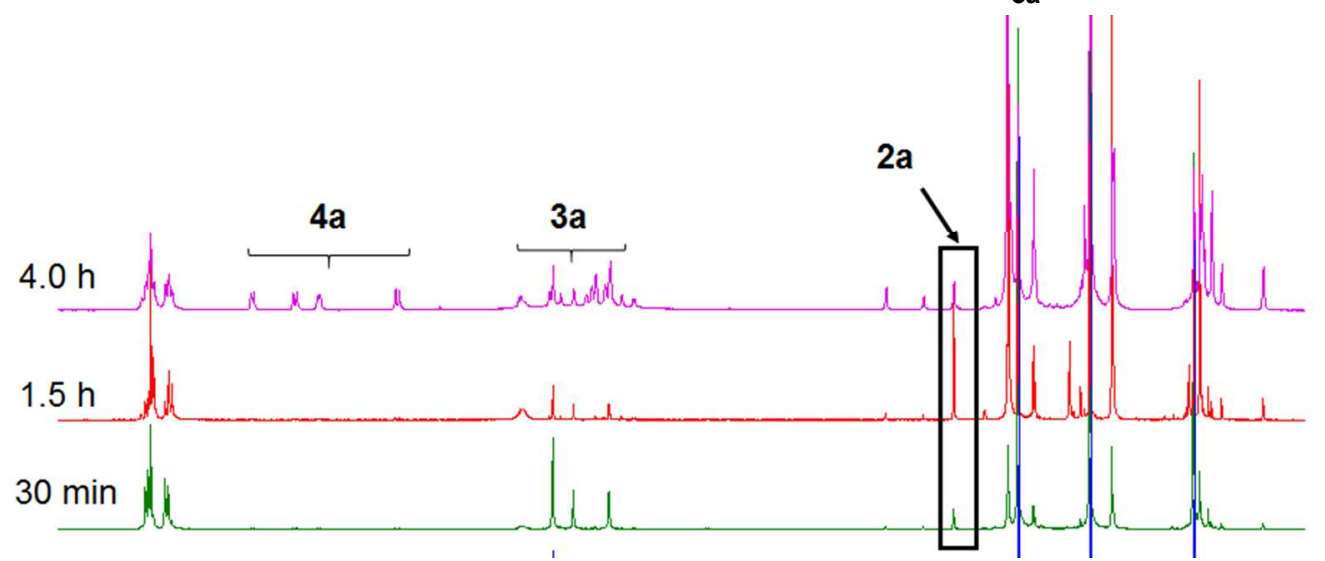

Figure S2. Intramolecular reaction of enoldiazoacetamide 1a catalyzed by $\mathrm{Cu}(\mathrm{MeCN})_{4} \mathrm{PF}_{6}$ through its cyclopropene intermediate $2 \mathbf{a}$ to form 3a and 4a monitored by ${ }^{1} \mathrm{H}$ NMR spectroscopy. 

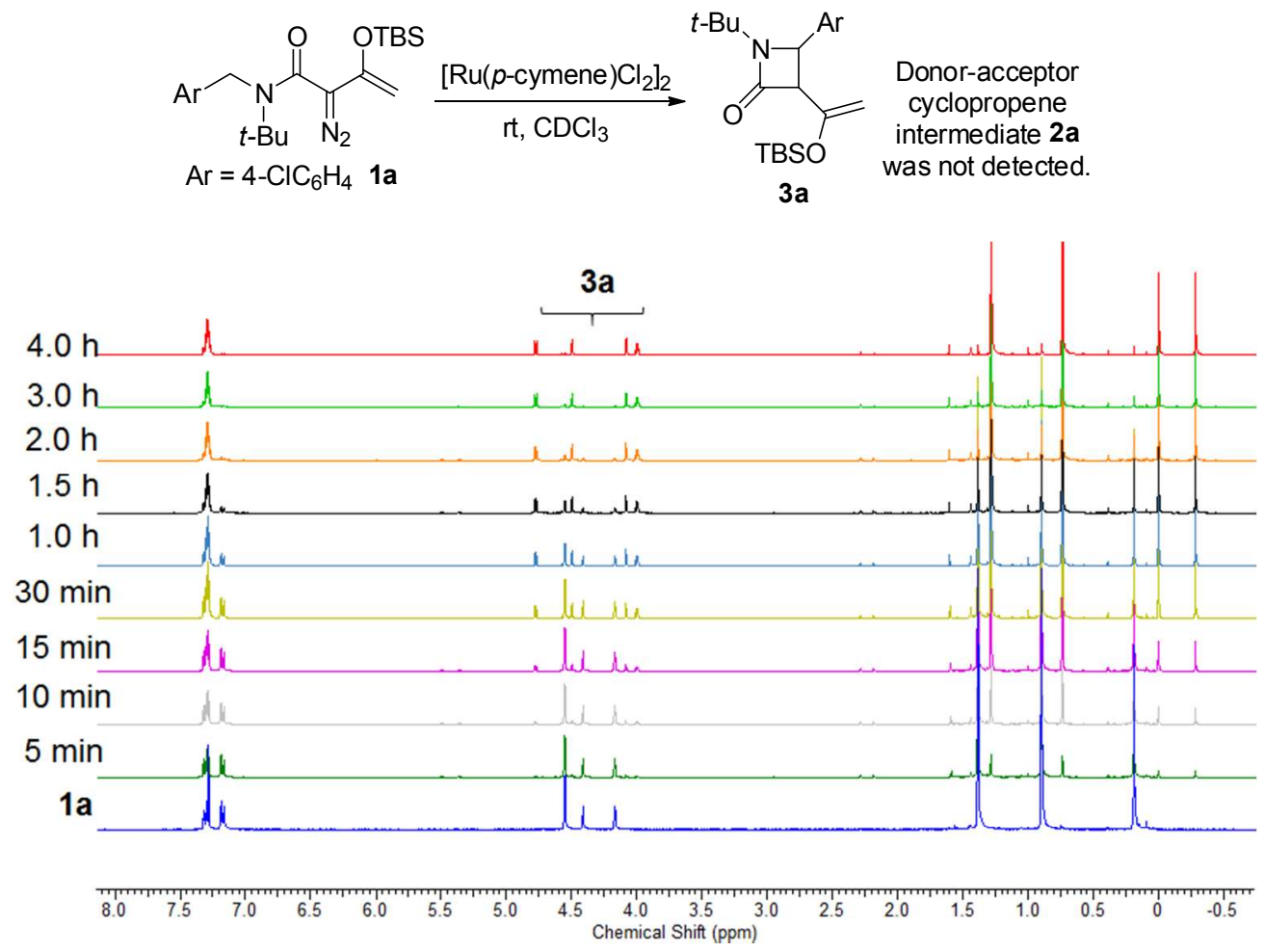

Figure S3. Intramolecular reaction of enoldiazoacetamide $\mathbf{1 a}$ catalyzed by $\left[\mathrm{Ru}(p \text {-cymene }) \mathrm{Cl}_{2}\right]_{2}$ to form $\mathbf{3 a}$ monitored by ${ }^{1} \mathrm{H}$ NMR spectroscopy.

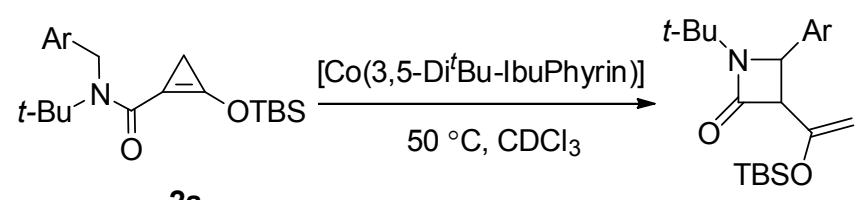

$2 a$

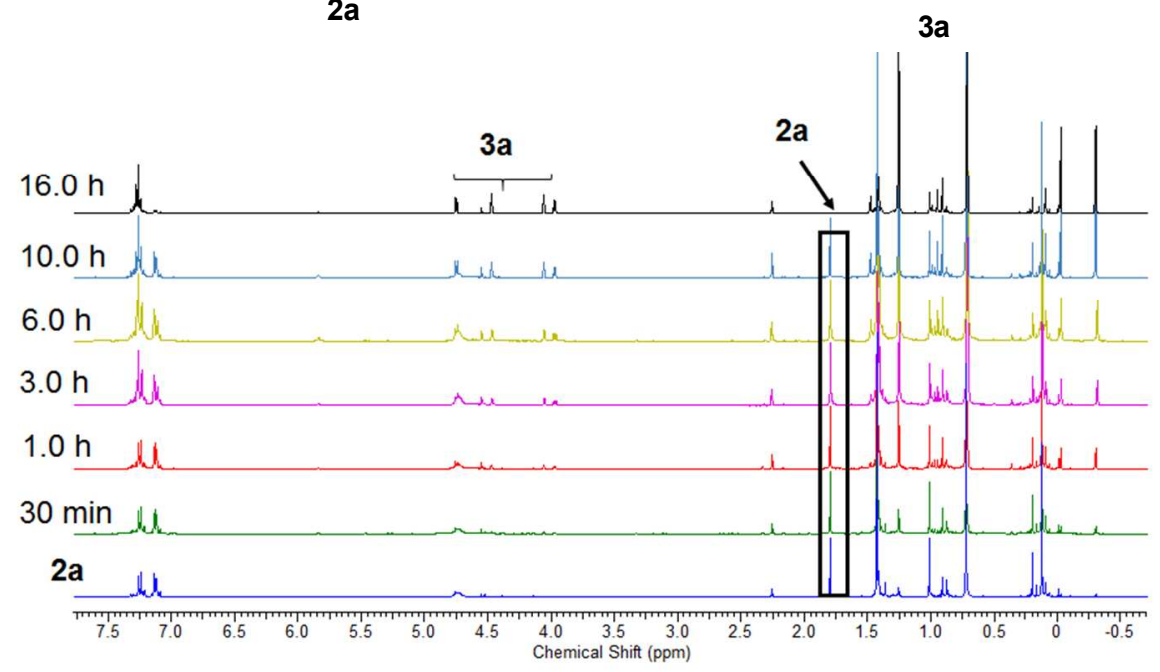

Figure S4. Intramolecular reaction of donor-acceptor cyclopropene 2 a catalyzed by [Co(3,5-Dit $\mathrm{Bu}-$ IbuPhyrin)] to form 3a monitored by ${ }^{1} \mathrm{H}$ NMR spectroscopy. 


\section{X-Ray Diffraction Analysis for Compound 6.}
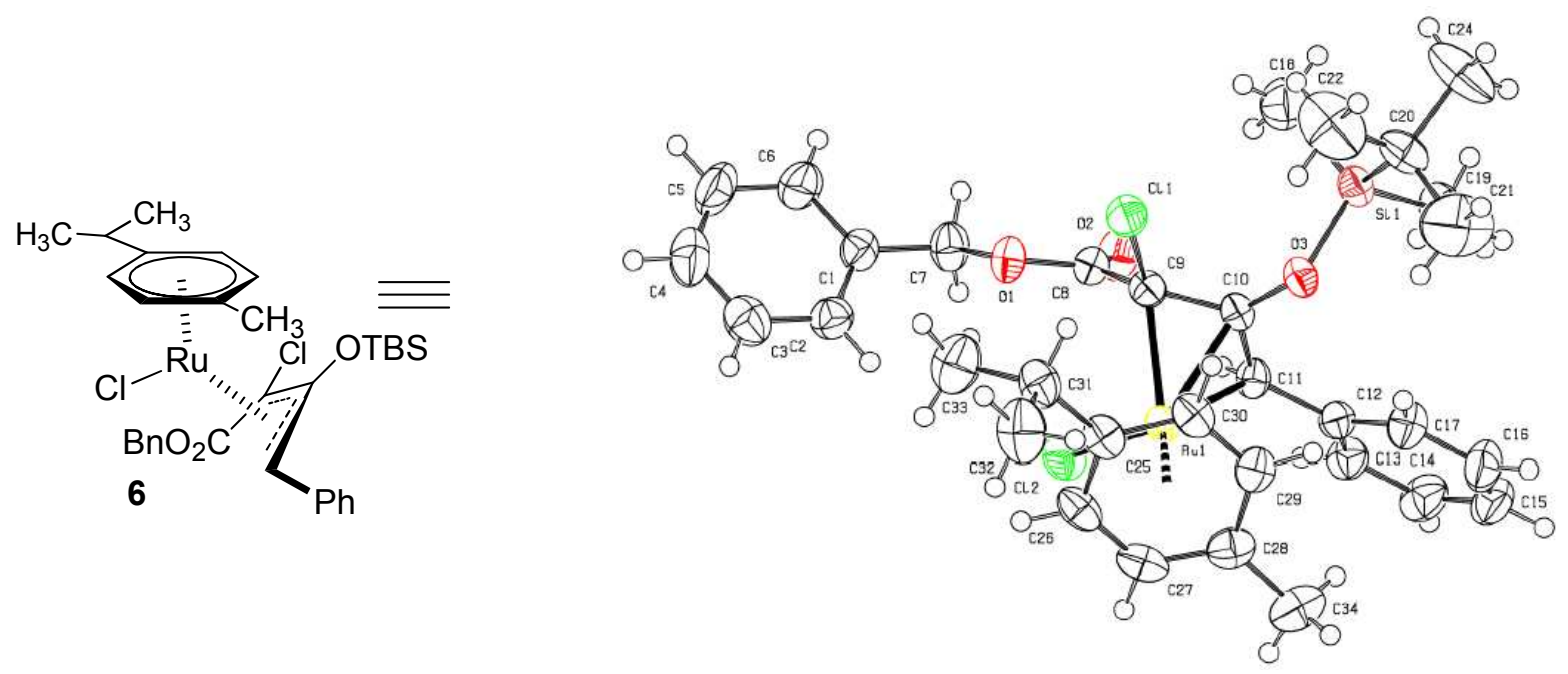

Figure S5. ORTEP view of 6. CCDC 1498257 contains supplementary crystallographic data for 6.

Single crystals of $\mathrm{C}_{33} \mathrm{H}_{42} \mathrm{Cl}_{2} \mathrm{O}_{3} \mathrm{RuSi} 6$ were prepared by slow evaporation of DCM. A suitable yellow plank-like crystal, with dimensions of $0.36 \mathrm{~mm} \times 0.26 \mathrm{~mm} \times 0.07 \mathrm{~mm}$, was mounted, using Paratone oil, onto a nylon loop. The data were collected at 98(2) K using a Rigaku AFC12 / Saturn 724 CCD fitted with MoK $\alpha$ radiation $(\lambda=0.71075 \AA)$. Data collection and unit cell refinement were performed using CrystalClear software. ${ }^{[1]}$ The total number of data were measured in the range $4.6^{\circ}<2 \theta<51.0^{\circ}$ using $\omega$ scans. Data processing and absorption correction, giving minimum and maximum transmission factors $(0.842,1.000)$, were accomplished with CrystalClear ${ }^{[1]}$ and $A B S C O R^{[2]}$, respectively. The structure, using Olex $2^{[3]}$, was solved with the ShelXT ${ }^{[4]}$ structure solution program using direct methods and refined (on $F^{2}$ ) with the ShelXL ${ }^{[5]}$ refinement package using full-matrix, least-squares techniques. All nonhydrogen atoms were refined with anisotropic displacement parameters. All hydrogen atom positions were determined by geometry and refined by a riding model.

$\left.{ }_{[1}^{1}\right]$ CrystalClear User's Manual, Rigaku/MSC Inc., Rigaku Corporation, the Woodlands, TX, 2011.

[2] T. Higashi, ABSCOR, Rigaku Corporation, Tokyo, Japan, 1995.

$\left.{ }^{3}\right]$ O. V. Dolomanov, L. J. Bourhis, R. J. Gildea, J. A. K. Howard, H. Puschmann, J. Appl. Cryst. 2009, 42, 339.

$\left[{ }^{4}\right]$ G. M. Sheldrick, Acta Cryst. 2015, A71, 3.

[5] G. M. Sheldrick, Acta Cryst. 2008, A64, 112. 
Table S1: Crystallographic data and structure refinement for CCDC 1498257.

\begin{tabular}{|c|c|}
\hline Identification code & CCDC 1498257 \\
\hline Empirical formula & $\mathrm{C}_{33} \mathrm{H}_{42} \mathrm{Cl}_{2} \mathrm{O}_{3} \mathrm{RuSi}$ \\
\hline Formula weight & 686.72 \\
\hline Crystal system & Monoclinic \\
\hline Space group & $P 12{ }_{1} / \mathrm{n} 1$ \\
\hline$a(\AA)$ & $10.1410(15)$ \\
\hline$b(\AA)$ & $15.406(2)$ \\
\hline$c(\AA)$ & $21.056(3)$ \\
\hline$\alpha\left({ }^{\circ}\right)$ & 90 \\
\hline$\beta\left(^{\circ}\right)$ & $95.288(3)$ \\
\hline$\gamma\left({ }^{\circ}\right)$ & 90 \\
\hline Volume $\left(\AA^{3}\right)$ & $3275.6(8)$ \\
\hline $\mathrm{Z}$ & 4 \\
\hline$\rho$ (calc.) & 1.393 \\
\hline$\lambda$ & 0.71075 \\
\hline Temp. (K) & 98(2) \\
\hline $\mathrm{F}(000)$ & 1424 \\
\hline$\mu\left(\mathrm{mm}^{-1}\right)$ & 0.709 \\
\hline $\mathrm{T}_{\min }, \mathrm{T}_{\max }$ & $0.842,1.000$ \\
\hline $2 \theta_{\text {range }}\left(^{\circ}\right)$ & 4.64 to 51.0 \\
\hline Reflections collected & 21737 \\
\hline Independent reflections & $\begin{array}{c}6095 \\
{[\mathrm{R}(\mathrm{int})=0.0664]}\end{array}$ \\
\hline Completeness & $99.7 \%$ \\
\hline Data / restraints / parameters & 6095 / 0 / 369 \\
\hline Observed data & 5512 \\
\hline
\end{tabular}




\begin{tabular}{|c|c|}
\hline$[\mathrm{I}>2 \sigma(\mathrm{I})]$ & 0.0973 \\
\hline$w R\left(F^{2}\right.$ all data $)$ & 0.0366 \\
\hline$R(F$ obsd data $)$ & 1.05 \\
\hline Goodness-of-fit on $F^{2}$ & $0.90 /-0.77$ \\
\hline largest diff. peak and hole $\left(\mathrm{e} \AA^{-3}\right)$ & \\
\hline
\end{tabular}

$$
\begin{gathered}
w R_{2}=\left\{\Sigma\left[w\left(F_{\mathrm{O}}^{2}-F_{\mathrm{c}}^{2}\right)^{2}\right] / \Sigma\left[w\left(F_{\mathrm{O}}^{2}\right)^{2}\right]\right\}^{1 / 2} \\
R_{1}=\Sigma|| F_{\mathrm{O}}|-| F_{\mathrm{c}} \| / \Sigma\left|F_{\mathrm{O}}\right|
\end{gathered}
$$


2-(tert-Butyl)-8a-[1-(tert-butyldimethylsilyloxy)vinyl]-2,3-dihydrocyclohepta[c]pyrrol-1(8aH)-one (4b).

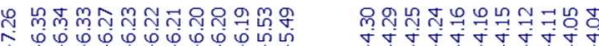
i<smiles>C=C(OC(C)(C)C)C12C=CC=CC=C1CN(C(C)(C)C)C2=O</smiles><smiles></smiles>

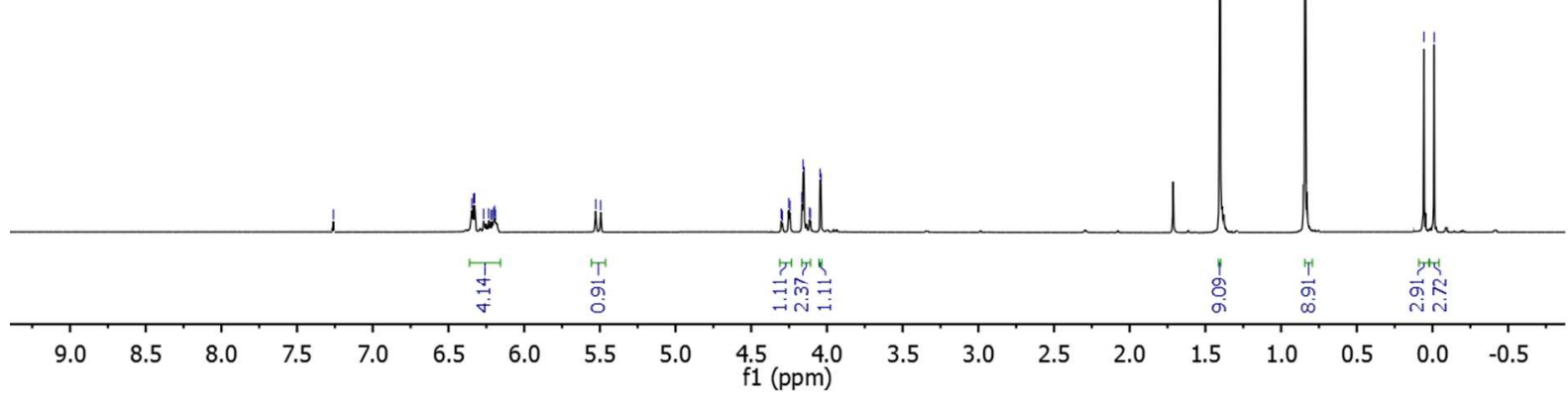

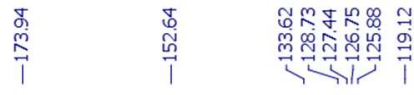<smiles>C=C(O[SbH3])C12C=CC=CC=C1CN(C(C)(C)C)C2=O</smiles>
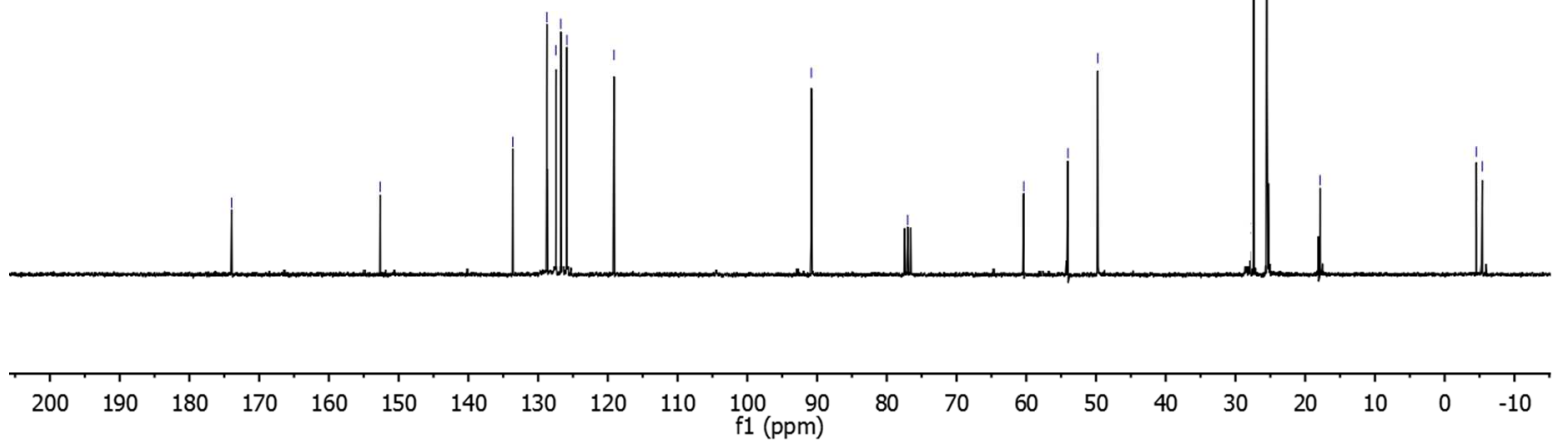
Methyl-4-1-(tert-butyl)-3-[1-(tert-butyldimethylsilyloxy)vinyl]-4-oxoazetidin-2-yl-benzoate (3d).

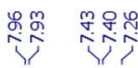

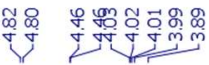

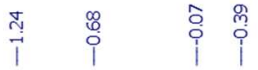<smiles>C=C(OC(C)(C)C)C1C(=O)N(C(C)(C)C)C1c1ccc(C(C)(C)C)cc1</smiles>

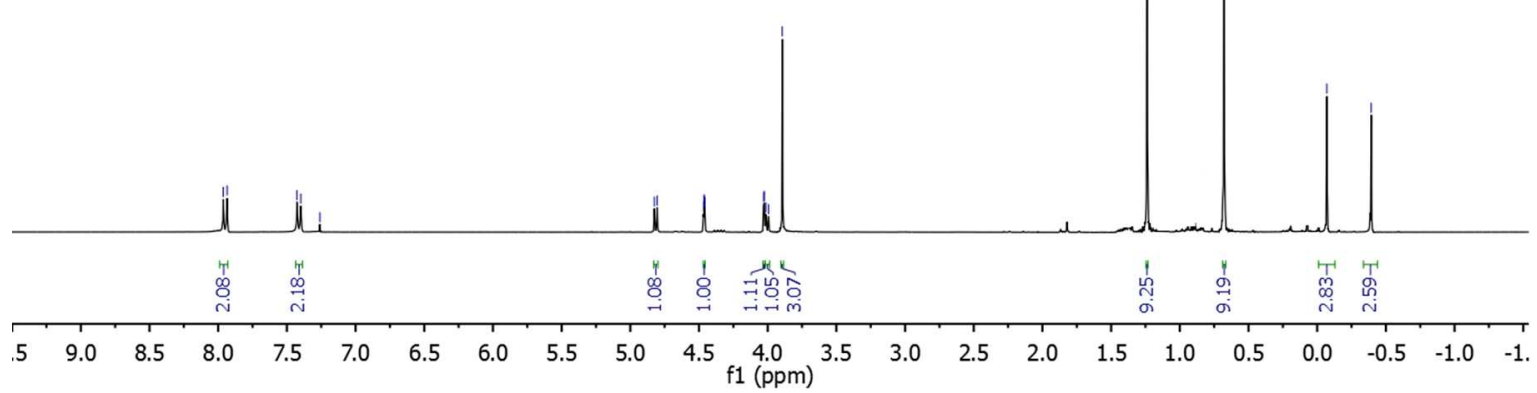

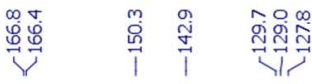

\%

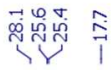<smiles>C=C(O[SnH3])C1C(=O)N(C(C)(C)C)C1c1ccc(C(=O)OC(C)(C)C)cc1</smiles> 
Methyl-2-(tert-butyl)-3a-[1-(tert-butyldimethylsilyloxy)vinyl]-3-oxo-1,2,3,3a-tetrahydrocyclohepta[c]pyrrole-6-carboxylate (4d)
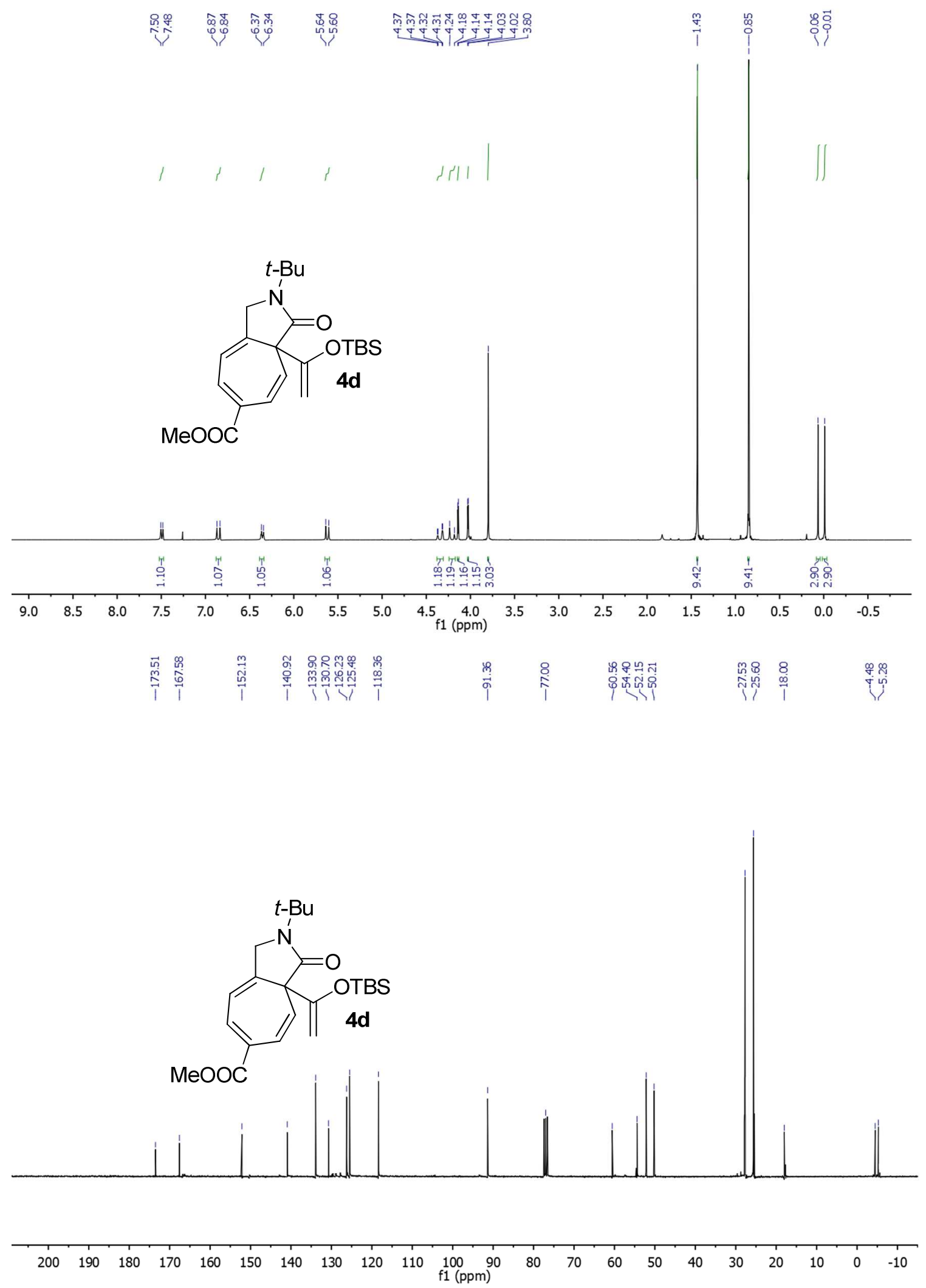
$\eta^{3}$-Allyl ruthenium(II) complex 6.

等
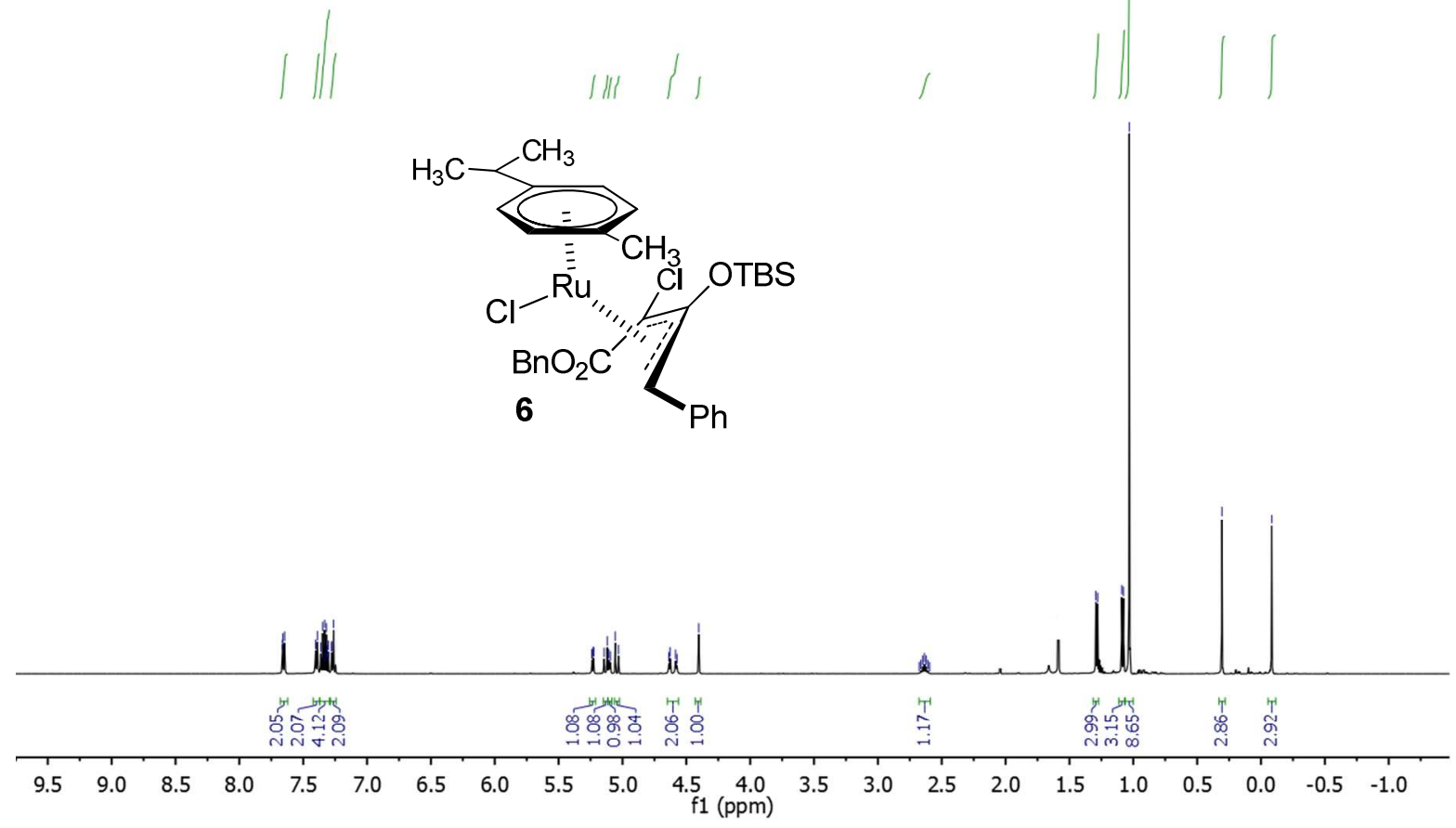

6

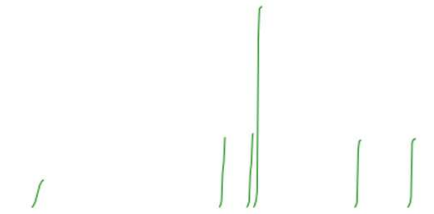

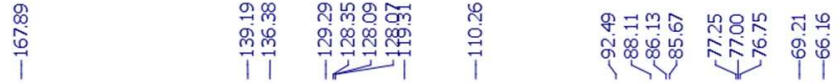
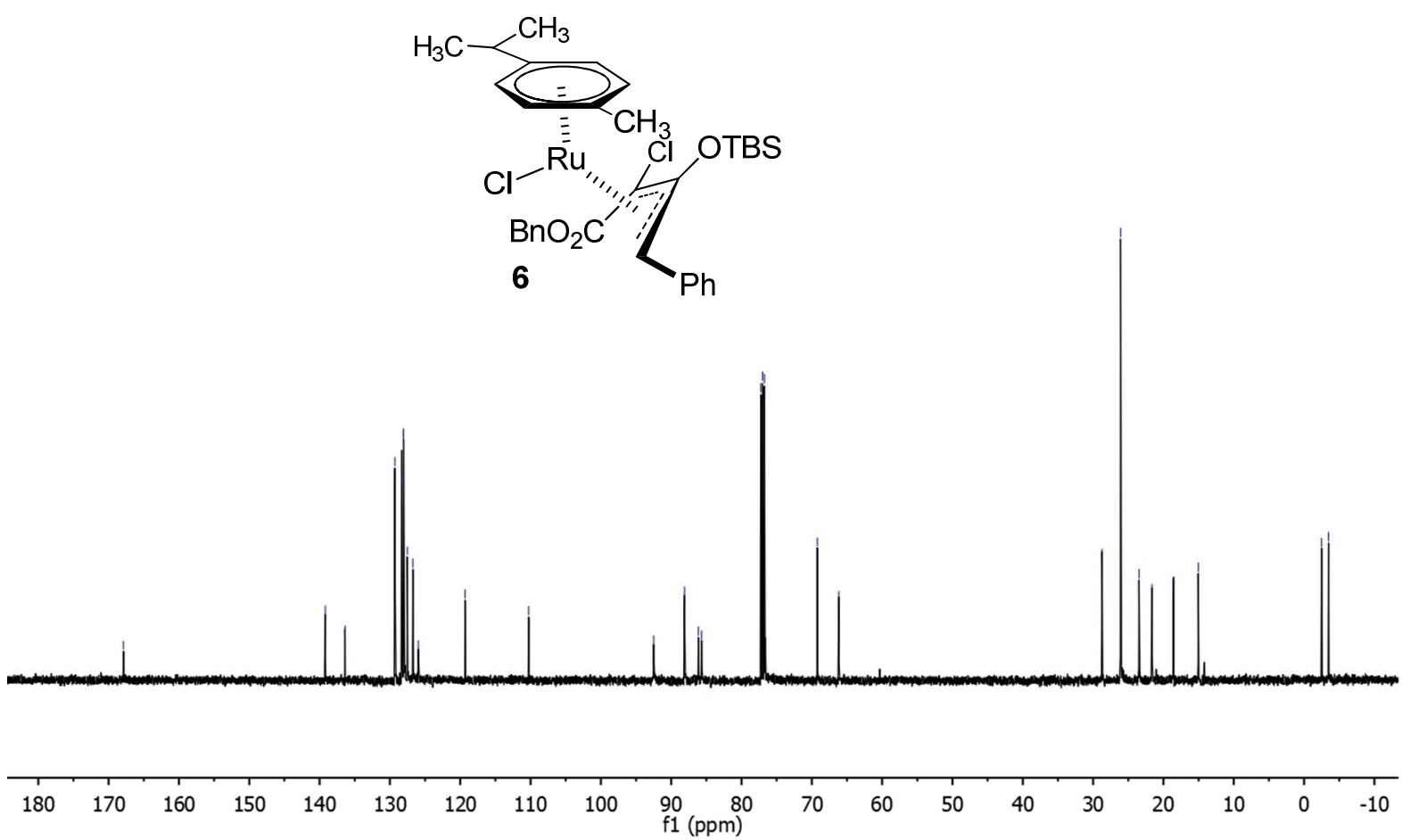